International Journal of Computer Networks \& Communications (IJCNC) Vol.3, No.5, Sep 2011

\title{
MODELS OF LINK CAPACITY DISTRIBUTION IN ISP'S ROUTER-LEVEL TOPOLOGY
}

\author{
Takahiro Hirayama, Shin'ichi Arakawa, Shigehiro Hosoki and Masayuki Murata \\ Graduate School of Information Science and Technology, \\ Osaka University, Osaka, Japan \\ \{t-hirayama, arakawa, s-hosoki, murata\} @ist.osaka-u.ac.jp
}

\begin{abstract}
Modeling the Internet is vital for network researches. Recent measurement studies on the Internet topology show that the degree distribution obeys the power-law distribution. However, only the degree distribution does not determine the performance of network control methods. As previous studies have shown, one of important factors to characterize the performance of network control methods in the Internet is a structure of topologies. However, other characteristics, which are even more important, are link capacities and node processing capacities of the network because these characteristics are particular to communication networks. In this paper, we investigate how to model the link capacity in the router-level topologies. We first reveal that the link capacity distribution of ISP's backbone network in Japan obeys a power-law with exponent -1.1. To clarify the reason for the link capacity distribution, we evaluate throughput of networks having various kinds of link capacity distributions. Our numerical results show that the network with power-law link capacity distribution can accommodate much more traffic than the network with exponential distributions of link capacities.
\end{abstract}

\section{KEYWORDS}

Power-law, Router-level Topology, Link Capacity, Modeling the Internet, Traffic Demand Matrix, Lognormal Distribution

\section{INTRODUCTION}

Modeling the Internet is one of important issues to evaluate network control methods [1]. Since the performance of network control methods strongly depends on Internet topology and capacity of links, a proper network model is necessary to show effectiveness of those methods. Therefore, it is important to reveal characteristics of a communication network and origin of them.

Measurement studies on the Internet topology show that the degree distribution obeys the power-law [2], [3]. That is, the existence probability $P(k)$ of node with $K$ out-going links approximates to $K^{r}(r$ is constant). There are many modeling methods for the power-law topology [4-6]. Reference [5] presents FKP model for a topology generation model that consider technical constraints of router-level topologies. However, the topology is much different from the actual ISP topologies [7]. In Ref. [6], the authors enumerate several topologies having the same degree distribution. Then, they evaluate the amount of traffic that the network can accommodate in each topology under the constraint of node processing DOI : $10.5121 /$ ijcnc.2011.3515 
International Journal of Computer Networks \& Communications (IJCNC) Vol.3, No.5, Sep 2011

capacities. The results indicate that the network throughput highly depends on the structure of topologies even though they have the same degree distribution.

Many researches about the modeling method of router-level topology have been discussed based on the characteristic that the degree distribution obeys the power-law. In these researches, there was an assumption that link capacities can be ignored, or they are identical. However, the performance of network strongly depends on link capacities because link capacities are characteristics particular to communication networks.

In this paper, we investigate the characteristic of link capacities in router-level topology. One of our main motivations in this paper is to investigate the modeling methodology for realistic ISP networks. For this purpose, characteristics particular to communication networks other than the degree distribution are important. First, we investigate the link capacity distribution in a Japanese ISP network using the capacity information presented in Ref. [8]. Results show that the link capacity distribution also obeys the power-law with exponent -1.11 . Second, to reveal the reason why the link capacity distribution obeys the power-law, we investigate the network performance under the constraint of link capacities. We generate three distributions of link capacities; power-law, exponential and identical. Then we assign the link capacities to ISP topologies, and compare the network performance. Results show that topologies with powerlaw link capacity distribution can accommodate more traffic than that with other two distributions. We also reveal that it is insufficient to consider only the constraint of node processing capacities, even though it is discussed in Ref. [6], in evaluating the network performance.

This paper is organized as follows. We introduce related work in Section 2. In Section 3, we investigate link capacity distribution in a Japanese ISP network. In Section 4, we explain about topology generation models and topologies which are used for evaluation. In Section 5, we investigate the difference of network performance by three distributions of link capacities. Section 6 concludes this paper.

\section{RELATED WORK}

There are relatively few studies on modeling router-level Internet topology [4-6, 9, 10]. Heckmann et. al. [9] present parameter settings for topology generator tools such as BRITE, TIERS, and GT-ITM to construct POP (point-of-presence)-level ISP topologies. Topology generation methods that consider technical constraints of router-level topologies are discussed in $[5,6]$. Reference [5] presents FKP model where a newly added node connects with existing nodes that minimize the sum of Euclidian distance between the nodes and logical distance from the existing node. The authors demonstrate that the degree distribution obeys the power-law with appropriate parameter settings. However, the topology generated by the model has too many nodes whose degree is one [11], and thus the topology is much different from the actual ISP topologies [7]. This is because ISP topologies are designed with technological, geographic, and business constraints $[12,13]$. In Ref. [6], the authors enumerate several topologies having the same degree distribution. Then, they evaluate the amount of traffic that the network can accommodate in each topology under the constraint of node processing capacities. The results indicate that the network throughput highly depends on the structure of topologies even though they have the same degree distribution. With the constraint of node processing capacities, a product of node degree and its corresponding link capacities is bounded. Thus, for maximize 
International Journal of Computer Networks \& Communications (IJCNC) Vol.3, No.5, Sep 2011

the traffic that the network can accommodate, non-hub nodes having small number of links connects with higher-capacity links, and hub-nodes having many links connects with lowercapacity links.

Moreover, many researchers have discussed about capacity distribution in power-law topologies [14-16]. Zhang et al. mentioned that node capacity distribution based on node betweenness centrality is appropriate to enhance network capacity [14]. Betweenness centrality is defined as the number of shortest paths that pass through the nodes or edges. In Ref. [15], Xia et al. assigned optimal node capacity with an optimization problem to maximize the system performance and the utilization ratio of capacity. This method provides a similar capacity distribution to that is allocated to be proportional to betweenness centrality of nodes in BA topologies. These researchers assumed that link capacity is unlimited. However, actual communication systems like the Internet have heterogeneous link capacity distribution. In Ref. [16], Ling et al. discussed about link capacity distribution in BA topologies. They claimed that link capacity distribution based on the product of both end nodes' degree maximizes network throughput. In these papers, model-based topologies are concerned. Model-based topologies

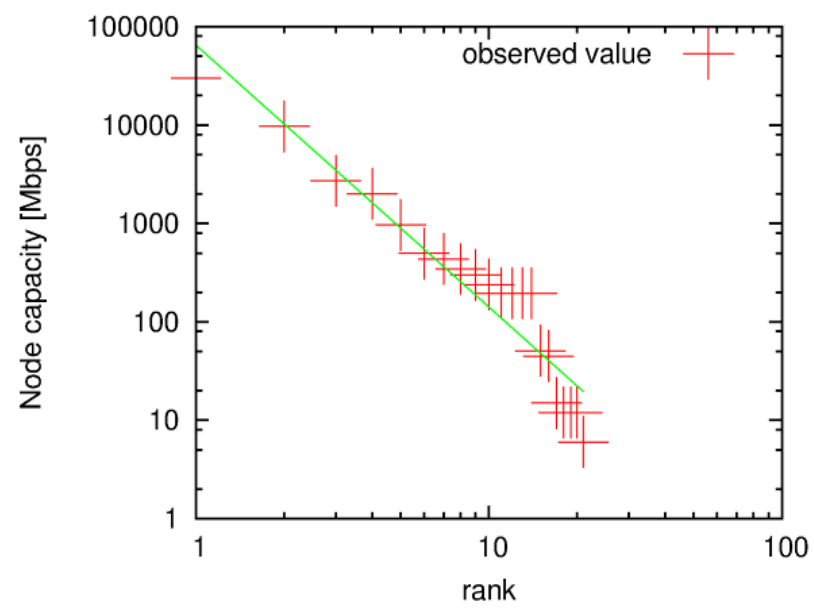

Figure 1: Node Processing Capacity Distribution in the IIJ Network

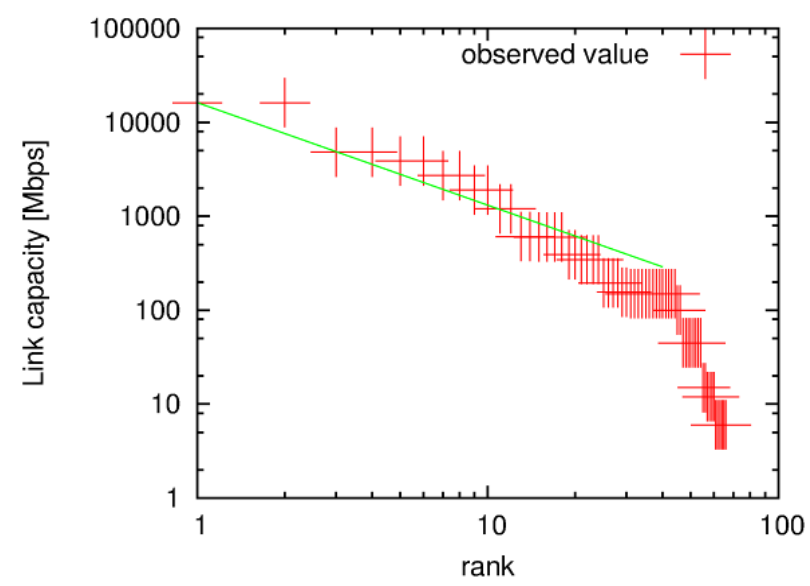

Figure 2: Link Processing Capacity Distribution in the IIJ Network 
International Journal of Computer Networks \& Communications (IJCNC) Vol.3, No.5, Sep 2011

have different properties from ISP topologies'. So, we evaluate the relationship between link capacity distribution and network throughput in ISP topologies.

\section{LINK CAPACITY DISTRIBUTION IN ISP ROUTER-LEVEL TOPOLOGY}

Ref. [8] investigates node processing capacities of a commercial ISP network in Japan. The author uses a disclosed information of link capacities in IIJ (Internet Initiative Japan) [17]. In the paper, a node processing capacity is defined as the sum of link capacity connected to the node. Figure 1, which was also presented in Ref. [8], shows the rank of node processing capacities in 2002. The vertical axis represents the node processing capacity and the horizontal axis represents the rank of that node. We observe that node processing capacity distribution obeys the power-law; its exponent is nearly -2.6 .

The result is interesting in that the power-law relationship again appears in the node processing capacity. However, one question arises whether the link capacity distribution follows the power-law or not. To answer this, we show the link capacity distribution in Figure 2; the result shows that the exponent of the link capacity distribution (for 40 higher-ranking) is -1 , i.e., the distribution obeys the zipf's law. The figure is obtained by using the information on IIJ backbone network in 2002, thus it is uncertain that other ISP topologies have the observation that the link capacity distribution obeys the zipf's law. However, we believe that the observation is general because we can easily derive it by the non-blocking configuration of routers. Supposing that there is one uplink port with the capacity of in a router and there are $\alpha$ numbers of links, then if the router to be non-blocking, the capacity of each link should be $X / \alpha$. With this case, the exponent of link capacity distribution becomes -1. Base of this discussion, in the next section, we investigate the performance of networks when the link capacity distribution obeys the power-law with exponent -1 .

\section{TOPOLOGIES FOR EVALUATION}

\subsection{ISP Topologies}

We prepare two ISP topologies; AT\&T and Sprint [18]. AT\&T topology has 523 nodes and 1304 links, and Sprint topology has 467 nodes and 1280 links. These topologies measured using the Rocketfuel tool [19].

\subsection{Abeline-inspired Topology}

As a router-level topology, we prepare the Abilene-inspired topology that is also evaluated in Ref. [6]. This topology is based on Abilene Network used for higher education. The Abileneinspired topology has 869 nodes and 877 links.

\subsection{BA Topology}

Barabasi and Albert proposed a BA model to generate topologies having a power-law degree distribution [4]. We call the BA topology as the topology generated by BA model. The BA model is characterized by two features: Incremental Growth and Preferential Attachment, The model starts with a topology with a small number of nodes and works as follows:

Step1 Make an initial topology that has mo nodes. 
International Journal of Computer Networks \& Communications (IJCNC) Vol.3, No.5, Sep 2011

Step2 Incremental Growth: Add a new node at each time step.

Step3 Preferential Attachment: Connect the new node to $m$ different nodes chosen with probability П:

$$
\prod\left(k_{i}\right)=\frac{k_{i}}{\sum_{j} k_{j}}
$$

Where $k_{i}$ is the degree of node $i$.

To follow this process, the topology with its degree distribution obeys the power-law is generated. In this paper, we generate BA topology to be the same number of nodes and links in Abilene-inspired topology. At first we use BA model with $m_{0}=1$, and generate 869 nodes and 868 links topology. Then, nine links are added based on rule of Step3. The topology with 869 nodes and 877 links is generated.

\subsection{FKP Topology}

The FKP model proposed by Fabrikant et al. [5] revealed that the power-law property of degree distribution can still be obtained by minimizing "distance" metrics. This model does not use preferential attachment to add links, and instead uses minimization-based link attachment. More specifically, the FKP model works as follows. Each new node arrives at randomly in the Euclidean space $\{0,1\}^{2}$. After arriving at new node $i$, the FKP model calculates the following equation for each node, $j$, already existing in the network: $\beta . w_{i j}+l_{o j}$ where $w_{i j}$ is the Euclidean distance (i.e., physical distance) between nodes and, and is the hop-counts distance between node and a pre-specified "root" node (node 0 ). $\beta$ is a parameter that weights the importance of physical distance. If $\beta$ has a lower value, each node tries to connect to higher degree nodes; $\beta=0$ is an extreme scenario that creates a star-topology. If $\beta$ has a higher value, each node tries to connect their nearest nodes. A topology with high a $\beta$ is shown to behaves like an ER topology. The power-law property of the degree distribution appears at a

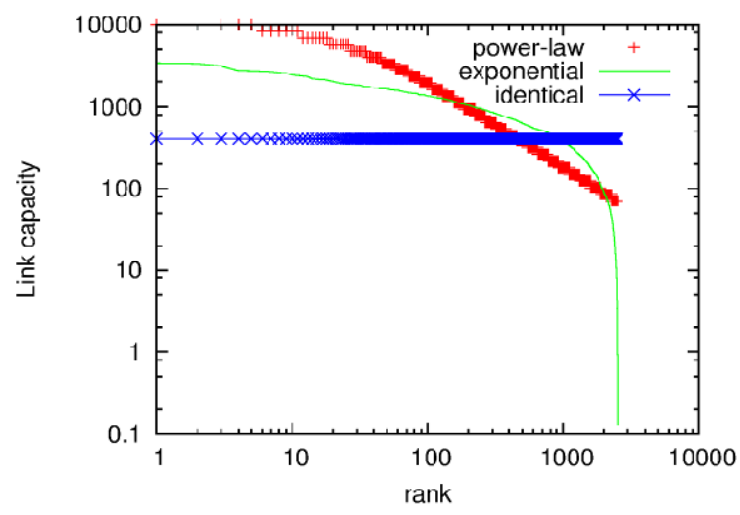

Figure 3: Normalized Link Capacity Distributions

moderate value of $\beta$ value. Here, there are several hub-nodes in each region, and the hub-nodes form a power-law. However, it is mentioned that the number of node whose degree is one is 
International Journal of Computer Networks \& Communications (IJCNC) Vol.3, No.5, Sep 2011

much more than that of ISP topology [6] and the degree distribution is different from that of ISP topology [18].

In this paper, we generate FKP topology to be the same number of nodes and links in Abileneinspired topology. For this purpose, we modify the FKP model; we first select nine nodes randomly before starting the topology generation and when each of these nine nodes is added, we generate two links between itself and a node satisfying the equation $\left(\beta . w_{i j}+l_{o j}\right)$, and another next node satisfying the equation. At last, a topology which has 869 nodes and 877 links is generated. )

\section{EVALUATION ON LINK CAPACITY DISTRIBUTION}

In Section 3, we show that the link capacity distribution of Japanese ISP obeys the power-law. For revealing the reason that link capacity distribution obeys power-law, we investigate network performance under the constraint of link capacities. In this paper, we define the network performance as the amount of traffic that the topology can accommodate. For comparison purpose, we generate three distributions of link capacities; power-law, exponential, and identical where all link capacities are identical. Then, the amount of traffic that the topology can accommodate for each distribution is compared.

\subsection{Link Capacity Distribution}

According to the observation in Section 3, we generate link capacity distribution that obeys power-law with exponent the -1 . We first prepare $m$ links with the capacity $X$ [bps]. Then, we prepare $\alpha \times m$ links each of which has $X / \alpha$ link capacity. By setting $m \leftarrow \alpha \times m$ and, $X \leftarrow X / \alpha$, we repeatedly prepare links. With this case, the exponent of link capacity distribution becomes -1 .

For comparison purpose, we generate other two link capacity distributions that obey exponential and identical. Figure 3 shows obtained distributions. The vertical axis represents the link capacity and horizontal axis represents the rank of that link. Note that we normalize the exponential and the identical distributions such that the sum of link capacities in each distribution is equaled. 
International Journal of Computer Networks \& Communications (IJCNC) Vol.3, No.5, Sep 2011

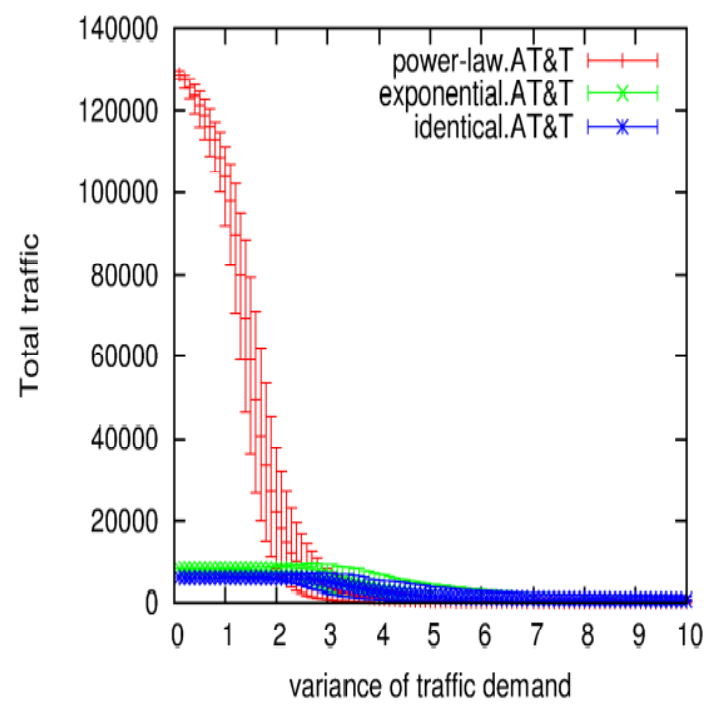

Figure 4: The Amount of Traffic that AT\&T

Topology can Accommodate

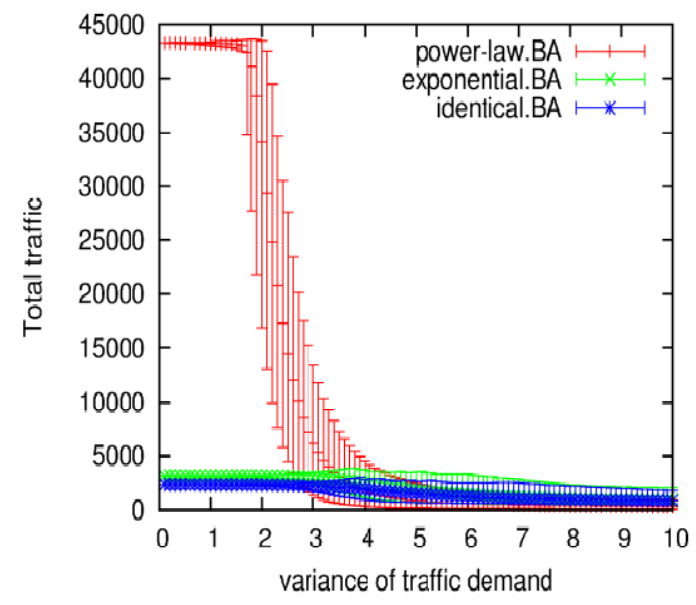

Figure 6: The Amount of Traffic that BA Topology can Accommodate

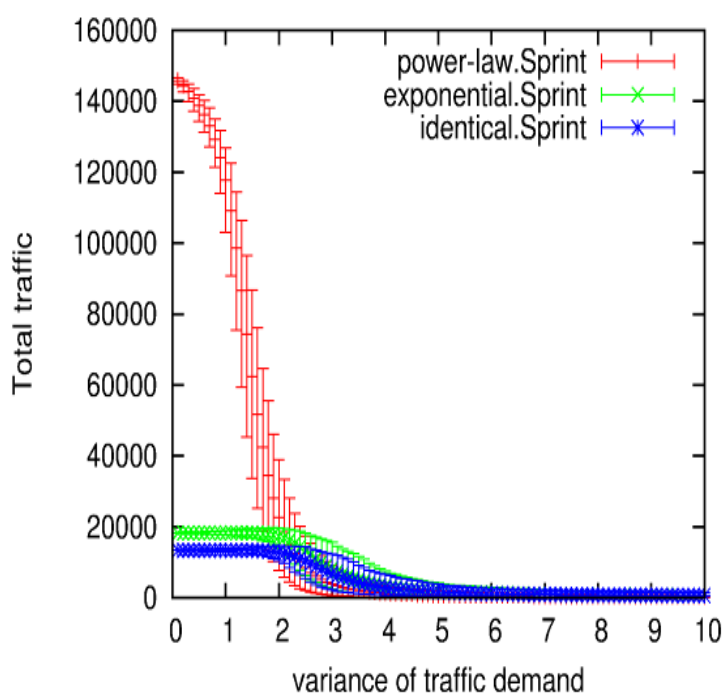

Figure 5: The Amount of Traffic that Sprint

Topology can Accommodate

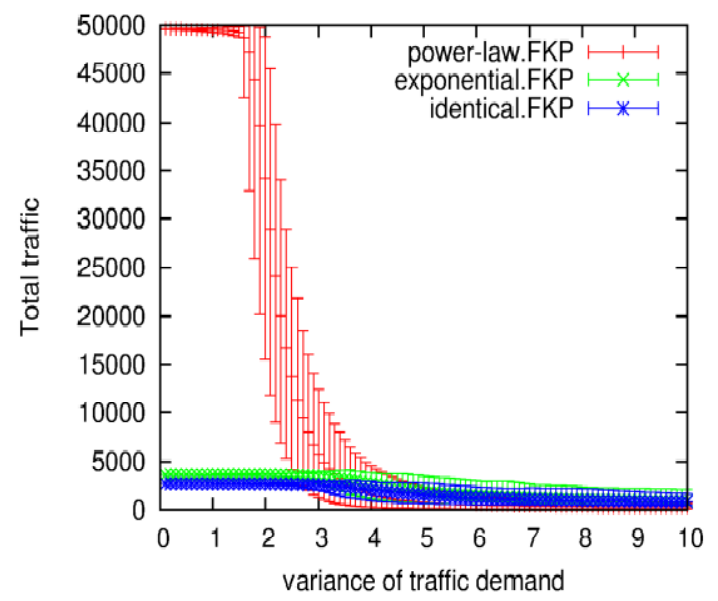

Figure 7: The Amount of Traffic that FKP Topology can Accommodate

\subsection{Assigning Link Capacity}

We then assign link capacity to each link in the topology. In this paper, we assume that the link capacity is assigned based on the edge betweenness centrality. More exactly, we assign link capacities in a descending-order to link with the edge betweenness centrality. The edge betweenness centrality is defined as the number of minimum hop paths that pass through the link. That is, the edge betweenness centrality represents the amount of contribution of a link to the communication in the network.

\subsection{Evaluation}

We evaluate the amount of traffic that each topology can accommodate under the constraint of link capacities. The traffic demand is generated by log-normal distribution since the distribution 
International Journal of Computer Networks \& Communications (IJCNC) Vol.3, No.5, Sep 2011

is known to be suitable for modeling the traffic demand [20]. We change the variance of the $\log$-normal distribution from 0.1 to 10.0 with the step 0.1 . The traffic demand is generated for each variance and sum of the traffic demands is normalized to 1. A minimum hop routing is applied to select the route of the traffic. Note that if there are several paths that have the same number of hops, one of them is selected randomly. The result is shown in Figure 4-8. The vertical axis represents the amount of traffic that the topology can accommodate and horizontal axis represents the variance of traffic demand. In these figures, $95 \%$ confidence interval is also presented.

In ISP topologies (Figure 4 and Figure 5), when the variance of traffic demand is less than 2, the topology with the power-law link capacity distribution can accommodate more traffic than

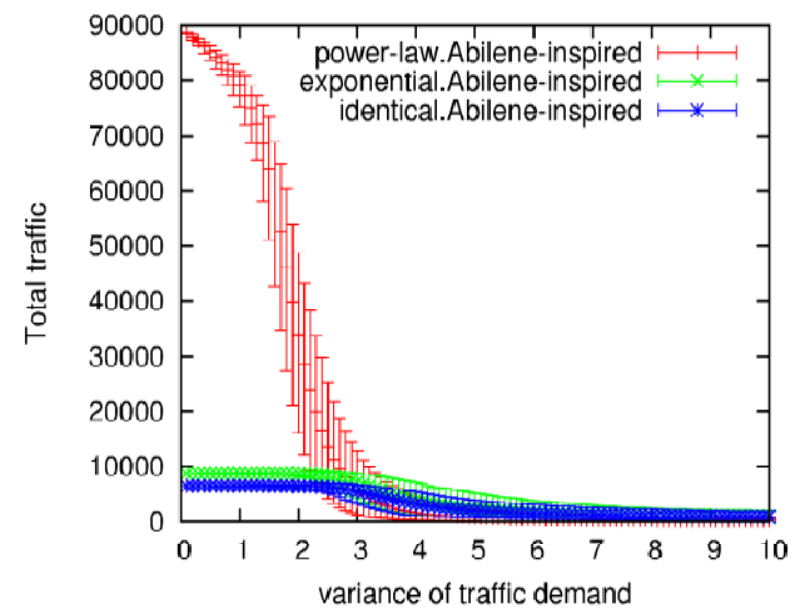

Figure 8: The Amount of Traffic that Abiline-inspired Topology can Accommodate

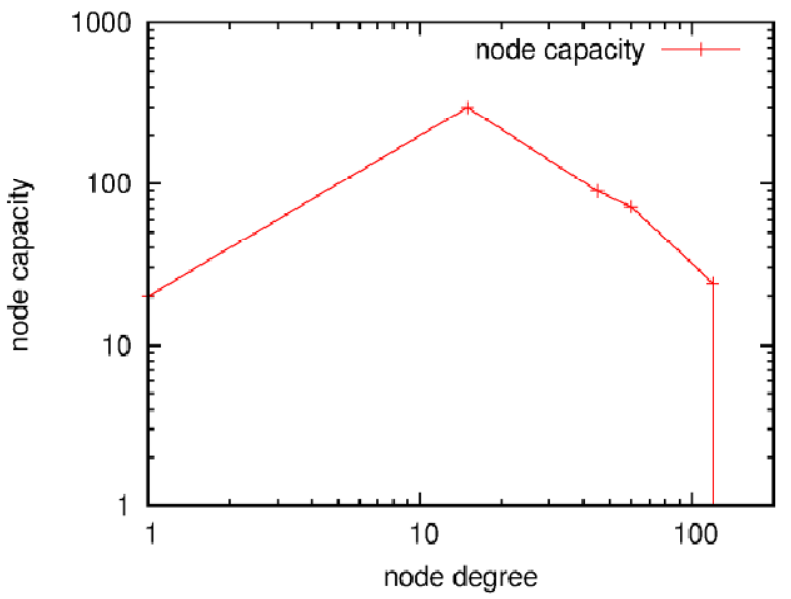

Figure 9: Relationship between Node Degree and Node Processing Capacity

topologies with other distributions. In Ref. [20], the variance of log-normal distribution is nearly 1 for fitting the observed traffic in real ISPs. Thus, in the realistic situation, the powerlaw link capacity distribution makes the topology more efficient. The topology with the powerlaw distribution accommodates the largest traffic among three distributions even though the 
International Journal of Computer Networks \& Communications (IJCNC) Vol.3, No.5, Sep 2011

variance of traffic demand changes around 1.0. However, the advantage of the power-law distribution decreases and diminishes when the variance is too large since elephant traffic appears in the traffic matrix. The same results are also observed in Figure 6, 7, 8. From the results of these figures, the power-law link capacity distribution can accommodates larger amount of the traffic than that of other distributions.

\subsection{Effects of Node Processing Constraint}

In Ref. [6], it is mentioned that the number of links connected to a router affects capacities of link. Figure 9 shows a relationship between node degree and node processing capacity. Due to the constraint of router technology, a product of node degree and its link capacities is bounded by the node processing capacity. That is, a router having few links can accommodate large capacity links, and a router having small capacity of links can accommodate many links. This section examines the difference of network performance between two constraints for the link capacities and the node processing capacities. We apply the relation in Figure 9 to the Sprint topology and Abilene-inspired topology. Figure 10 and Figure 11 show the amount of traffic that the topology can accommodate under the constraint of node processing capacities. For the comparison purpose, we again show the result of previous section in (a) of each figure and the result under the constraint of node processing capacities in (b) of each figure.

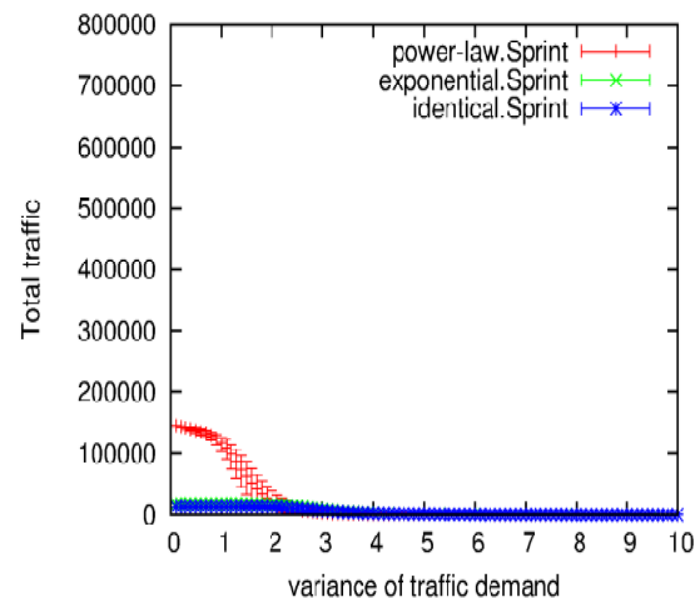

(a) The Case of Link Capacity Constraints

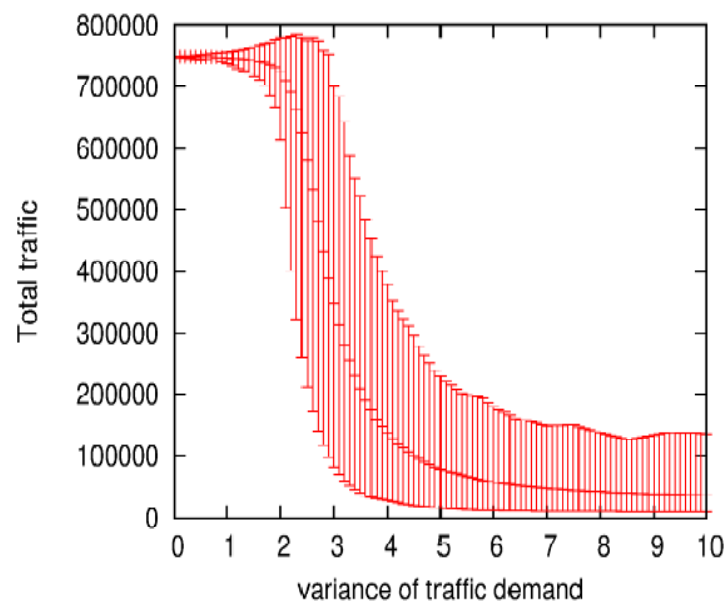

(b) The Case of Node Capacity Constraints

Figure 10: The Network Performance of Sprint Topology 
International Journal of Computer Networks \& Communications (IJCNC) Vol.3, No.5, Sep 2011

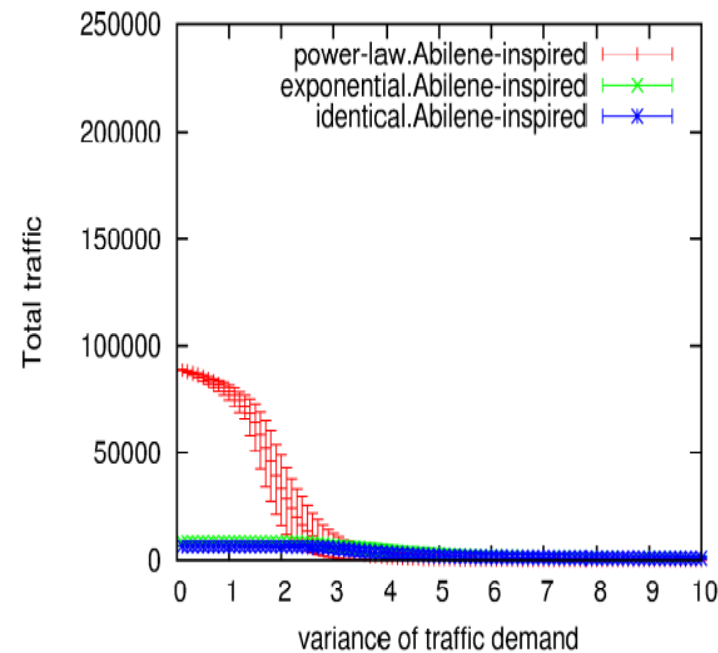

(a) The Case of Link Capacity Constraints

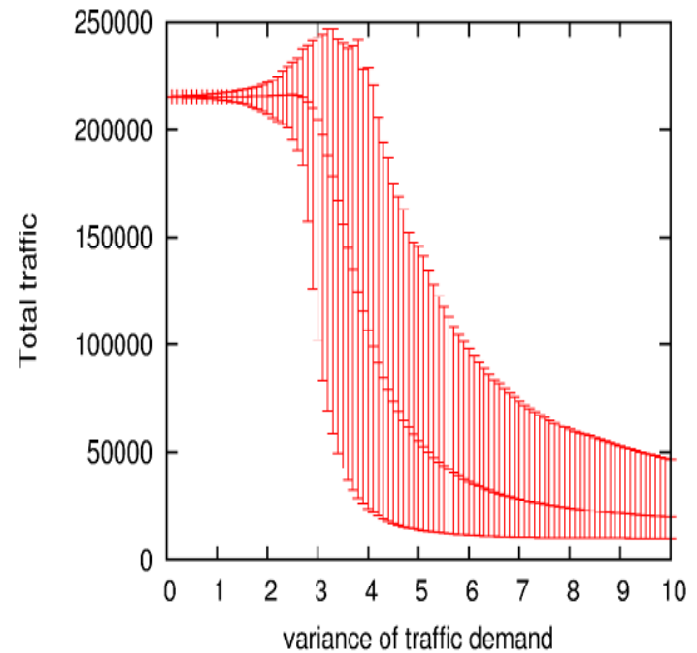

(b) The Case of Node Capacity Constraints

Figure 11: The Network Performance of Abiline-inspired Topology

Comparison between (a) and (b) of Figure 10 and Figure 11 shows the difference of amount of the traffic that the topology can accommodate with each constraint; the link capacities and the node processing capacities. These figures have a common tendency that amount of the traffic that the topology can accommodate gets smaller as the variance of traffic demand becomes larger. In other three topologies (BA topology, FKP topology and Abilene-inspired topology), the same tendency is observed. However, the amount of traffic that the topology can accommodate under the constraint of link capacities is strongly smaller than that under the constraint of node processing capacities. Thus, evaluation of network performance only the constraint of node processing capacities by its degree is inadequate for network evaluations.

\section{Conclusion}

Evaluation of the network control method needs proper model of the communication network. In this paper, we focused on link capacity in ISP router-level topology. First, we investigate link capacity distribution in Japanese ISP backbone network. Results show that link capacity distribution in ISP obeys the power-law. Next, for revealing the reason that link capacity distribution obeys power-law, we compared network performance with three link capacity distributions; power-law, exponential and identical. Results show that power-law link capacity distribution can accommodate large amount of the traffic. Moreover, under the constraint of only node processing capacities by its degree, amount of the traffic that the network can accommodate is over estimated. Thus to take characteristic that link capacity distribution obeys power-law into the modeling of router-level topology is important to evaluate the network control method properly.

\section{ACKNOWLEDGEMENT}


International Journal of Computer Networks \& Communications (IJCNC) Vol.3, No.5, Sep 2011

This research was supported in part by "Global COE (Centers of Excellence) Program" of the Ministry of Education, Culture, Sports, Science and Technology, Japan and "Grant-in-Aid for Scientific Research (A) 21240004" of the Japan Society for the Promotion of Science (JSPS) in Japan.

\section{REFERENCES}

[1] F. Sally and P. Vern, "Difficulties in simulating the Internet," IEEE/ACM Transactions on Networking, vol. 9, pp. 392-403, Feb. 2001.

[2] M. Faloutsos, P. Faloutsos, and C. Faloutsos, "On power-law relationships of the Internet topology," in Proceedings of ACM SIGCOMM'99, pp. 251-262, Oct. 1999.

[3] B. Zhang, R. Liu, D. Massey, and L. Zhang, "Collecting the Internet AS-level topology," ACM SIGCOMM Computer Communication Review, vol. 35, pp. 53-61, Jan. 2005.

[4] A. L. Barabási and R. Albert, "Emergence of scaling in random networks," Science, vol. 286, pp. 509-512, Oct. 1999.

[5] A. Fabrikant, E. Koutsoupias, and C. H. Papadimitriou, "Heuristically optimized trade-offs: A new paradigm for power laws in the Internet," in Proceedings of ICALP'02, pp. 110-122, July 2002.

[6] L. Li, D. Alderson, W. Willinger, and J. Doyle, "A first-principles approach to understanding the Internet's router-level topology," in Proceedings of ACM SIGCOMM'04, vol. 34, pp. 3-14, Oct. 2004.

[7] J. Alvarez-Hamelin and N. Schabanel, “An Internet graph model based on trade-off optimization," European Physical Journal B, vol. 38, pp. 231-237, Mar. 2004.

[8] H. Maruta, GLOCOM Review, vol. 9, pp. 1-16, Sep. 2003 (in Japanese). Available at page 10 of http://www.glocom.ac.jp/top/project/odp/library/76_01.pdf.

[9] O. Heckmann, M. Piringer, J. Schmitt, and R. Steinmetz, "Generating realistic ISP-level network topologies," IEEE Communications Letters, pp. 335-336, July 2003.

[10] S. Zhou, "Characterising and modelling the Internet topology - the rich-club phenomenon and the PFP model," BT Technology Journal, vol. 24, pp. 108-115, July 2006.

[11] N. Berger, B. Bollobás, C. Borgs, J. Chayes, and O. Riordan, "Degree distribution of the FKP network model," in Proceedings of ICALP'03, pp. 725-738, July 2003.

[12] D. Alderson, J. Doyle, R. Govindan, and W. Willinger, "Toward an optimization-driven framework for designing and generating realistic Internet topologies," ACM SIGCOMM Computer Communication Review, vol. 33, pp. 41-46, Jan. 2003.

[13] D. Alderson, L. Li, and W. Willinger, "Understanding Internet topology: principles, models, and validation," IEEE/ACM Transactions on Networking, vol. 13, pp. 1205-1218, Dec. 2005.

[14] G. Zhang, S. Zhou, D. Wang, G. Yan, and G. Zhang, "Enhancing network transmission capacity by efficiently allocating node capability,” Physica A, vol. 390, pp. 387-391, Jan. 2011.

[15] Y. Xia and D. Hill, "Optimal capacity distribution on complex networks," Europhysics Letters, vol. 89, p. 58004, Mar. 2010.

[16] X. Ling, M. Hu, W. Du, R. Jiang, Y. Wu, and Q. Wu, "Bandwidth allocation strategy for traffic systems of scale-free network," Physics Letters A, vol. 374, pp. 4825-4830, Nov. 2010.

[17] “Internet Initiative Japan,” http://www.iij.ad.jp/. 
International Journal of Computer Networks \& Communications (IJCNC) Vol.3, No.5, Sep 2011

[18] R. Fukumoto, S. Arakawa, T. Takine, and M. Murata, "Analyzing and modeling router-level Internet topology,” in Proceedings of ICOIN’07, pp. 171-182, Jan. 2007.

[19] N. Spring, R. Mahajan, D. Wetherall, and T. Anderson, "Measuring ISP topologies with Rocketfuel," IEEE/ACM Transactions on Networking, vol. 12, pp. 2-16, Feb. 2004.

[20] A. Nucci, A. Sridharan, and N. Taft, "The problem of synthetically generating IP traffic matrices: Initial recommendations," ACM SIGCOMM Computer Communication Review, vol. 35, pp. 19-32, July 2005 .

\section{Authors}

Takahiro Hirayama received a M.S. degree from Osaka University in 2010, where he is currently a doctoral student in Information Science and Technology. His research interest includes complex networks.

Shin'ichi Arakawa received M.E. and D.E. degrees in informatics and mathematical science from Osaka University in 2000 and 2003. He is currently an Assistant Professor at the Graduate School of Information Science and Technology, Osaka University. His research interests include optical networks and complex networks. He is a Member of IEEE.

Shigehiro Hosoki received a M.S. degree from Osaka University in 2011. His research interest includes complex networks.

Masayuki Murata received M.E. and D.E. degrees in information and computer sciences from Osaka University in 1984 and 1988. In April 1984, he joined the Tokyo Research Laboratory IBM Japan as a Researcher. From September 1987 to January 1989, he was an Assistant Professor with the Computation Center, Osaka University. In February 1989, he moved to the Department of Information and Computer Sciences, Faculty of Engineering Science, Osaka University. From 1992 to 1999, he was an Associate Professor with the Graduate School of Engineering Science, Osaka University, and since April 1999, he has been a Professor. He moved to the Graduate School of Information Science and Technology, Osaka University in April 2004. He has published more than 300 papers in international and domestic journals and conferences. His research interests include computer communication networks, performance modeling, and evaluation. He is a Member of IEEE, the Association for Computing Machinery (ACM), The Internet Society, and IPSJ. 